Voix et Images

volxetimages

\title{
Une saison dans la vie d'Emmanuel : le discours du conte
}

\section{Elaine D. Cancalon}

Volume 15, numéro 1 (43), automne 1989

Jacques Poulin

URI : https://id.erudit.org/iderudit/200820ar

DOI : https://doi.org/10.7202/200820ar

Aller au sommaire du numéro

\section{Éditeur(s)}

Université du Québec à Montréal

\section{ISSN}

0318-9201 (imprimé)

1705-933X (numérique)

Découvrir la revue

\section{Citer cet article}

Cancalon, E. D. (1989). Une saison dans la vie d'Emmanuel : le discours du conte. Voix et Images, 15(1), 102-110. https://doi.org/10.7202/200820ar d'utilisation que vous pouvez consulter en ligne.

https://apropos.erudit.org/fr/usagers/politique-dutilisation/ 


\section{Une saison dans la vie d'Emmanuel: le discours du conte}

\section{par Elaine D. Cancalon, Florida State University}

On sait l'importance qu'a eue la publication, en 1965, d'Une saison dans la vie d'Emmanuel de Marie-Claire Blais. Comme Gabrielle Roy qui, vingt ans auparavant, dans Bonheur d'occasion, avait révélé la misère de la grande ville, Marie-Claire Blais peint une condition de vie tout aussi dure, dans un petit village à la campagne. Il est vrai que Gabrielle Roy avait brisé l'écran idyllique derrière lequel la littérature dissimulait sa perception de la véritable condition du peuple. Sur le plan formel pourtant, elle ne s'était guère détachée de la tradition réaliste européenne: un narrateur extradiégétique fait filtrer son récit par diverses focalisations: on voit ce que les personnages voient; on sait ce qu'ils pensent et on entend leurs dialogues, mais ils ne parlent jamais directement au lecteur. Tout est toujours transmis par la voix du narrateur. L'intérêt d'Une saison dans la vie d'Emmanuel provient par contre non seulement de la critique sociale, mais aussi de l'originalité des procédés discursifs.

Dans Esthétique et théorie du roman, Mikhail Bakhtine démontre que le roman surtout est fait du plurilinguisme des discours, puisant leur origine dans la vie complexe du peuple:

[le] roman et les genres littéraires en prose se sont constitués dans le courant des forces décentralisatrices et centrifuges [...] en bas, sur les tréteaux des baraques et des foires, résonnait le plurilinguisme du bouffon [...] se déroulait la littérature des fabliaux et des soties, des chansons des rues, des dictons et des anecdotes. ${ }^{1}$

Bakhtine explique pourquoi toute forme romanesque est, de par sa nature même, intertextuelle:

[Tous les langages du plurilinguisme] peuvent se placer sur le plan unique du roman, qui peut rassembler les stylisations parodiques des langages des divers genres, différents aspects de stylisation et de présentation des langages professionnels engagés et ceux des générations, les dialectes sociaux et autres [...] Tous peuvent être attirés par le romancier pour orchestrer ses thèmes et réfracter l'expression (indirecte) de ses intentions et jugements de valeur. ${ }^{2}$

1 Mikhail Bakhtine, Esthétique et théorie du roman, Paris, Gallimard, 1978, p. 96.

2 Ibid., p. 113. 
Les discours présents dans un roman sont donc étroitement liés à l'évolution sociologique d'un peuple et traduisent la complexité (en termes bakhtiniens, le dialogisme) de cette évolution.

Les définitions de l'intertextualité que Julia Kristeva propose se rapprochent étroitement des idées de Bakhtine. Le phénomène du mélange des genres est repris par Kristeva dans le Texte du Roman où elle en démontre l'importance sociologique:

La fin du moyen âge, tout en mettant en valeur la phonétique en introduisant dans le texte culturel l' espace (bourgeois) de la foire, du marché, de la rue, se caractérise également par une pénétration massive du texte écrit: le livre cesse d'être un privilège de nobles et d'érudits et se démocratise. ${ }^{3}$

Mais ce qui nous intéresse aussi dans le propos de Kristeva, c'est l'idée que tout texte représente un moment de l'histoire où le réseau infini des intertextes qu'il contient se stabilise, ne fût-ce que provisoirement:

Le texte littéraire actuellement traverse la face de la science, de l'idéologie et de la politique comme discours [...] Pluriel, plurilinguistique parfois, et polyphonique souvent (de par la multiplicité de types d'énoncés qu'il articule), il présentifie le graphique de ce cristal qu'est le travail de la signifiance prise à un point précis de son infinité: un point présent de l'histoire où cette infinité insiste. ${ }^{4}$

Nous pourrons donc interpréter le roman de Marie-Claire Blais comme un point présent de l' histoire où cette infinité insiste, car l'œuvre est faite d'un enchevêtrement de genres qui semblent tous demander le droit de s'exprimer. Et la question posée (comment faire équivaloir discours et pouvoir?) est le problème précis du Québec, mais aussi une des questions capitales de l'histoire des sociétés.

L'originalité d'Une saison dans la vie d'Emmanuel vient donc de la multiplicité des discours qui, comme nous le verrons, révèlent une préoccupation fondamentale du peuple québécois: la prise de la parole. Mais on verra aussi qu'il y a une mise en question des formes traditionnelles et un effort pour les retravailler afin d'exprimer les préoccupations d'une société nouvelle. La première structure que l'on rencontre dans le roman est l'archétype du conte oral (conte de fées, conte des veillées). La manifestation principale de cette forme, qui ouvre le roman et lui donne sa base formelle, est aussi l'expression d'une famille «matricentrique» qui traduit déjà un des conflits de la société québécoise. S'il est vrai que faire régner l'ordre relève de l'autorité paternelle (tradition qui provient des sources européennes du peuple), il y a dans le Nouveau Monde une certaine fonction dominatrice de la mère au sein de la famille, qui est une conséquence des conditions de travail de l'homme «pionnier» s'aventurant sur

3 Julia Kristeva, le Texte du roman, La Haye/Paris, Mouton, 1970, p. 147.

4 Julia Kristeva, Sèméiôtikè. Recherches pour une sémanalyse, Paris, Seuil, 1969. p. 16. 
de nouvelles terres, ou bien occupé à défricher pour «faire de la terre». Dans Une saison dans la vie d'Emmanuel, cette domination est exprimée à travers la prise de la parole. La première voix que l'on entend est celle de la Grand-Mère Antoinette qui raconte les événements de la vie au nouveau-né Emmanuel. Une remarque d'Anatole France dans «Le dialogue sur les contes de fées» résume bien l'importance de la fonction narrative de l'aïeule qui remonte aux Contes de Ma Mère l'Oie:

Qu'est-ce que Ma Mère l'Oie, sinon notre ä̈eule à tous et les aïeules de nos aïeules, femmes au cour simple, aux bras noueux, qui firent leur tâche quotidienne avec une humble grandeur et qui, desséchées par l'âge, n'ayant comme les cigales, chair ni sang, devisaient encore au coin de l'âtre, sous la poutre enfumée et tenaient à tous les marmots de la maisonnée ces longs discours qui leur faisaient voir mille choses? 5

Cette forme archétypale est transformée dans le roman par le jeu des voix narratives. La parole sera passée, non d'une aïeule à l'autre, mais de l'aïeule directement à l'enfant. Dans la tradition orale, la parole est signe de la sagesse qui est un attribut de l'âge. S'il est vrai que l'ouverture du roman respecte cette tradition en donnant la parole à la grand-mère, la suite la dément en passant la parole à Jean Le Maigre. Un renversement est donc accompli selon l'équation suivante:

(réalisme)

(rêve) vieillesse matricentrique traditionnelle

vs

enfance mâle prodigieuse

La transformation ainsi produite fait remplacer un archétype par un autre: le mythe de l'enfant voyant rimbaldien remplace celui de l'aïeule sage. La sagesse change de sexe, de forme et d'âge. Les allusions intertextuelles à Rimbaud sont claires; à commencer par le titre même du roman qui aurait très bien pu être Une saison dans la vie de Jean Le Maigre. Nous verrons plus loin le rôle des autres rimbaldismes et le rapport entre Jean Le Maigre et Emmanuel.

Que ce soit le domaine de la grand-mère ou celui de Jean Le Maigre, la puissance et la sagesse sont l'apanage de la parole. Les autres personnages (le père, la mère, Héloïse, les autres enfants) ont à peine le droit de prononcer quelques paroles et certains demeurent totalement muets. Aucun n'est doué d'une voix narrative.

Examinons d'abord la fonction de la grand-mère et ensuite le rôle joué par l'enfant prodige. Le roman s'ouvre sur une description de la Grand-Mère Antoinette dominant le petit Emmanuel qui voit d'abord ses pieds, symboles de majesté: nobles et pieux [...] l'image sombre de l'autorité et de la patience ${ }^{6}$. On

5 Anatole France, le Livre de mon ami, Paris, Calmann-Lévy, 1885, p. 289-290.

6 Marie-Claire Blais, Une saison dans la vie d'Emmanuel, Montréal, Stanké, 1965 , p. 7. Toutes les citations sont tirées de cette édition. Le numéro de la page paraîtra entre parenthèses. 
voit déjà ici le rôle de l'intertextualité dans l'allusion à Berthe aux grands pieds. La focalisation du nouveau-né ${ }^{7}$ accentue dès la première scène l'opposition entre l'aieule et l'enfant. Emmanuel voit à peine sa grand-mère mais il entend déjà sa voix. En effet, Antoinette prend la parole dès le début et, jalouse de sa puissance, tente de faire taire l'expression déjà révoltée d'une nouvelle génération: $\mathrm{Ne}$ crie pas, de quoi te plains-tu donc? Ta mère est retournée à la ferme. Tais-toi jusqu'à ce qu' elle revienne. Ah! déjà tu es égoüste et méchant, déjà tu me mets en colère! (p. 7-8) Si Emmanuel doit se taire, c'est pour mieux écouter sa grand-mère qui exprime son désir de domination par la parole. Quoique la première focalisation appartienne au bébé, la voix narrative est celle de l'aïeule qui le force à écouter l'exposé des difficultés de la vie qui l'attend. Ainsi le rôle d'Emmanuel se confond avec celui du lecteur, narrataires tenus en laisse par la voix de l'autorité: Mais Grand-Mère Antoinette se croyait immortelle. Toute sa personne triomphante était immortelle aussi pour Emmanuel qui la regardait avec étonnement: «Oh! mon enfant, personne ne t'écoute, tu pleures vainement, tu apprendras vite que tu es seul au monde! (p. 10, c'est moi qui souligne.)

Mais aussi, le discours de la grand-mère a pour fonction de renseigner le lecteur. L'exposition du premier chapitre est accomplie par le récit d'Antoinette qui usurpe, pour ainsi dire, le rôle du narrateur omniscient et utilise l'enfant comme transmetteur. Sa puissance est ainsi traduite par sa maitrise de la parole, tout narrateur étant en même temps créateur.

Cette parole est aussi exercée comme pouvoir sur les membres de la famille. Les êtres qui entourent la grand-mère se fondent dans un mutisme anonyme. Ni la mère ni le père ne possèdent de nom. Ils sont donc réduits à leur fonction biologique. La mère est condamnée au silence le plus absolu. Pas une seule fois dans tout le roman on n'entend sa voix. Et ce silence est souvent rappelé:

Sa mère est silencieuse. Elle sera toujours silencieuse. (p. 13)

Sa mère, elle, ne dit rien, ne répond plus [...] elle semblait vouloir dire quelque chose, mais elle ne disait rien. (p. 15)

7 Cette utilisation du nouveau-né comme instance focalisatrice, au début et à la fin du roman, illustre parfaitement la distinction utile faite par Genette entre le voir (focalisation) et le dire (narration). Comme l'explique Genette, celui qui parle ne se confond pas toujours avec celui qui voit: $[. .$.$] la plupart des travaux théoriques sur$ ce sujet $[. .$.$] souffrent à mon sens d^{\prime}$ une fâcheuse confusion entre ce que j'appelle ici mode et voix, $c^{\prime}$ est-à-dire entre la question quel est le personnage dont le point de vue oriente la perspective narrative? et cette question tout autre: qui est le narrateur? - ou pour parler plus vite, entre la question qui voit et la question qui parle? (Figures III, Paris, Seuil, 1972, p. 203). Or, le choix du nouveau-né tranche nettement la question. Emmanuel ne peut pas parler. Ses pensées et ses sensations ne peuvent qu'être devinées et verbalisées par un autre, et cet autre est le narrateur hétérodiégétique. Aucun narrataire ne recevra jamais la parole d'Emmanuel - à moins qu'ayant grandi, lui aussi apprenne à parler comme son frère. Mais cela est un problème à résoudre dans une autre fiction. 


\section{[...] mais comme aujourd' hui elle écoutera, ne dira rien. (p. 17)}

Sa mère esquissait parfois un geste, un signe imperceptible de défaillance ou de pitié [...]. (p. 22)

Quant au père, il parlera par phrases coupées et sera le plus souvent désigné par l'anonymat de son sexe (la voix de l'homme). Sa fonction familiale, loin de s'exprimer par une parole autoritaire, est réduite à une sorte de bestialité inconsciente (il bat ses enfants, ou les jette dehors, et viole sa femme):

La voix d' homme n' est qu' un murmure. Elle se perd, disparait. (p. 13)

Il y a aussi les orphelinats, dit la voix de l' homme. (p. 14)

Mais il y a quelqu'un dont la grand-mère accepte l'autorité et c'est parce que, comme elle, il est gardien de la parole. C'est Jean Le Maigre. L'association entre Antoinette et ce demier résulte de leur croyance en la force et la nécessité du langage. Si on sait que la grand-mère a été la première à apprendre à lire, c'est parce que Jean Le Maigre nous le dit: quelle maladie apprendre à lire, à la fin, c'est ma grand-mère qui l'apprit la première (p. 74). La première apparition du jeune poète nous le révèle caché sous la jupe de l'aïeule (vieux lieu commun), un livre à la main: Mon Dieu, il y a quelqu' un sous ma robe [...] Ou étais-tu encore? Tu lisais sous la table? (p. 16) Jean devient en effet son livre:

- Tu as tort de rire, dit le père, je peux te l'arracher des mains, ce livre.

Jean Le Maigre secoue la tête, il montre son front blanc sous les cheveux:

- Il est trop tard, j'ai lu toutes les pages. On ne peut pas brûler les pages que j' ai lues. Elles sont écrites là! (p. 19)

Dans cet enfer où vit l'enfant, la grand-mère fait aussi fonction de muse protectrice: Viens près de moi [...] on ne peut pas te faire du mal quand tu es près de moi [dit-elle]. (p. 20) Le génie vivant en pleine misère et dont les poux qui l'assaillent sont signe des démons qu'il faut exorciser pour que son talent s'exprime, est un vieux mythe ${ }^{8}$ déconstruit par Jean Le Maigre dans un poème

8 Des motifs semblables se retrouvent dans le folklore des Amérindiens. Selon Stith Thompson, The Folktale in a Primitive Culture, New York, Holt, Rinehart \& Winston, 1946, p. 343, Almost everywhere are found stories of the defeat of a monster by lousing his head. D'autres légendes remplacent les poux par des serpents, mais l'acte est souvent interprété comme une libération. L'anecdote suivante est racontée par Thomas R. Henry dans Wilderness Messiah, New York, William Sloane, 1955, p. 37:

The thoroughly converted Hiawatha agreed to undertake the mission [conversion of the cannibal monster Ododarhoh]. In some way he broke through the thick shell of ancient evil which had grown around the nucleus of ancient good in the monster's soul... He scombed the serpents - evil and insane thoughts - which grew out of the friend's skull until they became strands of ordinary hair. From this exploit he 
en prose qui pourrait être tiré des Illuminations, œuvre qui, elle aussi, exprime des visions originales à travers la déconstruction des vieux mythes:

Dès ma naissance, j'ai eu le front couronné de poux! Un poète, s'écria mon père, dans un élan de joie. Grand-Mère, un poète! Ils s'approchèrent de mon berceau et me contemplèrent en silence. Mon regard brillait déjà d' un feu sombre et tourmenté. Mes yeux jetaient partout dans la chambre des flammes de génie. "Qu'il est beau, dit ma mère, qu'il est gras, et qu'il sent bon! Quelle jolie bouche! Quel beau front!» Je bâillais de vanité, comme j'en avais le droit. Un front couvert de poux et baignant dans les ordures! Triste terre! Rentrées des champs par la porte de la cuisine, les Muses aux grosses joues me voilaient le ciel de leur dos noirci par le soleil. Aie, comme je pleurais, en touchant ma tête chauve... (p. 66)

Alors que le poème de Rimbaud, «Les chercheuses de poux» 9 , reprend les vieux thèmes romantiques de la souffrance du poète et de l'action bienfaitrice des Muses, le passage tiré de l'autobiographie de Jean Le Maigre met en question ces mêmes mythes, grâce à un ton ironique et à une recombinaison cocasse d'éléments: clichés romantiques, le poète au front "couronné» ici de poux plutôt que de laurier; éléments stéréotypés des contes de fées: Ils s'approchèrent de mon berceau; tropes du poète voyant: Mes yeux jetaient partout dans la chambre des flammes de génie; figure comique des Muses qui ne sont plus, comme chez Rimbaud, deux grandes sours charmantes, mais des filles qui travaillent aux champs, des Muses aux grosses joues [qui...] voilaient le ciel de leur dos noirci par le soleil.

Le passage de la parole de la grand-mère à celle de Jean Le Maigre représente donc une transformation plus profonde qu'une simple reprise de la parole par la jeunesse. Le renversement va plus loin que l'équation présentée plus haut et voudrait démontrer, paradoxalement, qu'une certaine innocence passive chez les ancêtres sera remplacée par un cynisme actif et qui se voudrait durable chez les jeunes. Ce passage est démontré de plusieurs façons. La parole de la grand-mère est orale et presque inconsciente. Antoinette raconte des histoires qui s'intègrent à sa vie quotidienne. Elle commence à peine à se rendre compte de l'importance de sa parole qui ne se libère jamais totalement du cadre du récit premier: c'est-à-dire que le narrateur extradiégétique est toujours présent derrière les discours de la grand-mère. Elle ne se voit pas, ne se décrit pas: elle est toujours focalisée à travers l'autre (Emmanuel ou le narrateur).

received his real name, Ayonwartha, which means «he-who-combs or "he, the comber». He was a pionneer psychiatrist. [Je voudrais remercier Tim Barton et Greg Toole de Florida State University pour la recherche qu'ils ont faite sur ce sujet.]

La déconstruction intertextuelle d'Une saison n'est donc pas limitée à ses sources européennes.

9 Arthur Rimbaud, Pages choisies, Paris, Larousse, 1972, p. 28 (Nouveaux Classiques Larousse). 
Par contre, lorsque Jean Le Maigre prend la parole, son récit métadiégétique remplace le récit premier et en demeure totalement indépendant pendant trentecinq pages. C'est une autobiographie écrite qui subsistera après sa mort. Là où la grand-mère révèle l'innocence des ancêtres dans ses descriptions de la vie quotidienne, et dans sa croyance absolue en la valeur des vieilles traditions, comme à celles de l'Église, Jean s'amuse à déconstruire tous les vieux mythes et formes de discours qu'il recombine pour former des fables burlesques. Tout pour Jean est prétexte à l'invention créatrice: les jeux sexuels auxquels il se livre avec son frère sont interrompus de poèmes, d'histoires étranges (p. 50); la visite nocturne à Héloïse se transforma en roman (p. 52). Ainsi, la sexualité même est déconstruite et démystifiée car le secret et l'interdiction des fantasmes de masturbation sont convertis en expression littéraire.

L'autobiographie de Jean Le Maigre est une reprise complexe d'un nombre considérable d'intertextes de la tradition européenne. Il serait trop long ici de passer en revue toutes les formes de discours qui sont reprises et déconstruites en même temps par ce jeune poète ${ }^{10}$. Examinons-en pourtant quelques-unes:

- Le conte de fées: a) comme Hansel et Gretel, les jeunes enfants sont abandonnés par [leur] mère, orphelins errants au visage barbouillé de soupe et au derrière cuit par les coups [...] (p. 73). Dans le conte, on sait que les enfants doivent être cuits et devenir la soupe! b) la description de ses jours en prison se transforme en un conte de fées où ogres et monstres divers peuplent le monde: - Qu'allez-vous faire de lui, monsieur, il est jeune, très jeune [...] - Nous allons le manger pour dessert, et garder ses os pour jouer aux billes. (p. 92) Mais cette figure aussi est satirisée, car ayant parlé des ogres qui dévorent les petits enfants, les dernières phrases de Jean mourant exprimeront le regret de perdre la faim: Le plus triste, c'est que moi qui étais si gourmand, j'ai soudain perdu l'appétit. (p. 98)

— Les allusions à Dante et à Rimbaud: tout le long de l'autobiographie, il est question d'une descente aux enfers. Mais ce voyage symbolique, dont le but est d'arriver à la connaissance de soi, est converti par Jean et son frère en la joie presque sensuelle qu'apporte la pyromanie: Nous avons mis le feu partout, misérables oisifs que nous étions. (p. 73)

10 On peut interpréter cet élément de reprise et de déconstruction selon l'extension que donne Kristeva au concept bakhtinien du carnavalesque:

Le roman a emprunté au carnaval aussi cette tendance à dévaloriser le texte qui le précède et qui, de par le fait de son antériorité, est devenu la loi du genre [...] Faut-il mentionner tous les nombreux exemples de la littérature qui dialogue avec les textes précédents pour les relativiser? La parodie des romans chez Cervantes [...] la ridiculisation $[. .$.$] des$ romans romantiques chez Flaubert, des mythes grecs chez Joyce? Le roman semble de tout temps avoir voulu se faire comme une OPPOSITION à une loi qui n' est pas seulement celle du genre, mais aussi celle - idéologique - du discours de son époque, et cette opposition est la marque même de la participation du texte romanesque à l'histoire. (Julia Kristeva, Texte du Roman, op. cit., p. 175-176) 
- La remise en question de tous les mythes romantiques qui entourent la vie d'un poète: Jean se moquera surtout du mythe du poète tuberculeux. Resté jovial jusqu'à ses dernières paroles, il rejette l'idée selon laquelle souffrance égale génie. Tout ascétisme lui est étranger et son attachement tenace à la vie sensuelle durera jusqu'à sa mort.

Peut-on dire qu'il y a une allégorie du passage de la parole de la naïveté des vieux au cynisme des jeunes qui traduit l'évolution de la pensée au Québec? La destruction des vieux mythes accomplie par la nouvelle génération, par quoi sera-t-elle remplacée? Où sont les créateurs des nouveaux mythes nécessaires à tout peuple?

Comme si la voix du génie devait se taire à jamais, la narration homodiégétique s'arrête avec la mort de Jean Le Maigre et la voix narrative redevient celle de l'anonymat. Les autres personnages n'auront plus le droit de raconter leur propre aventure: même l'histoire d'Héloïse, qui constitue quand même une révolte importante, est transmise par un narrateur extradiégétique. Nous ne pouvons pas, comme nous l'avons fait avec Jean, vivre simultanément avec elle ses fantasmes car ils nous sont décrits de l'extérieur. Le même procédé est utilisé pour la narration des aventures de Pomme (qui se coupe la main à l'usine) et du Septième (qui est la proie innocente des avances du frère Théodule). Tout se passe comme si cette jeune génération, privée de la parole, ne peut que se laisser manipuler par une société qui les exploite et où ils n'auront aucun pouvoir: Le Septième [...] finirait sans doute en prison, comme lui avait dit son père, tant de fois. (p. 175)

Mais il reste peut-être encore de l'espoir, car la parole de Jean Le Maigre est préservée par ses écrits et par la lecture qu'en fait sa grand-mère. Le récit accomplit ainsi un retour cyclique, car c'était Antoinette qui la première avait appris à lire. Maintenant c'est l'aïeule qui subira une transformation grâce à l'œuvre de l'enfant: Grand-Mère Antoinette eût voulu serrer contre son cœur ces pages, afin que chacune s'inscrive en elle pour toujours avec sa morsure fraîche, son secret féroce. (p. 111) Le rapport entre la grand-mère et l'enfant devient encore plus intime après la mort de celui-ci car, alors que vivant il devenait son livre (On ne peut pas brûler les pages que $j$ 'ai lues. Elles sont écrites là! [p. 19]), mort, c'est son livre qui devient le poète: Elle se penchait encore sur Jean Le Maigre vivant, puisqu' elle lisait ses auvres [...] (p. 123) Il y a donc identité parfaite entre la parole et la vie.

Vers la fin du roman, la grand-mère Antoinette reprend la parole dans ses dialogues avec Emmanuel. Il y a pourtant une certaine ambiguïté dans le sens symbolique de ce dénouement qui pose des questions auxquelles le roman n'apporte pas de réponse définitive. «Emmanuel» signifie celui qui a été choisi par Dieu, ce qui suggérerait (comme la parole est adressée à lui) la présence de la divinité. Et pourtant son frère vient de tout remettre en question et même le rôle de la religion. Dans quelle mesure alors cet enfant devra-t-il se sacrifier comme le Christ pour qu'une nouvelle croyance soit instaurée? À quoi servira donc la transmission de la parole à Emmanuel? La nouvelle génération parlerat-elle avec la force et le savoir nécessaires pour se sortir de la misère du silence 
(l'enfer)? Osera-t-elle enfin se servir de cette seule arme efficace? La faim d'Emmanuel peut très bien symboliser le désir de connaissance, mais les dangers qu'il implique sont rappelés par Antoinette qui, malgré son autorité au sein de la famille, comprend que la violence exercée par le mâle peut l'emporter sur la sagesse: [Jean] voulait tout savoir, le pauvre enfant. Il en est mort. Son père l'a trop battu. Toi aussi tu seras battu si tu poses des questions. Vaux mieux te taire et aller couper du bois comme les autres. (p. 137) Est-ce que les jeunes Québécois de 1965 allaient donc être toujours battus par leurs «pères» (c'est-à-dire l'autorité politique) pour le péché de vouloir savoir et parler? Allaient-ils être condamnés à une saison en enfer?

Mais c'est le printemps et Emmanuel se lève joyeux en voyant le soleil. Cette chaleur aussi peut être symbolique de la lumière future, car Jean le Maigre était, comme Rimbaud, un voyant. La dernière phrase du livre présente les deux points de vue d'une manière équilibrée:

[1] Oui, ce sera un beau printemps, disait Grand-Mère Antoinette, mais [2] Jean Le Maigre ne sera pas avec nous cette année... (p. 175)

Il fera beau mais celui qui possédait la parole n'est plus là. Sa mission serat-elle accomplie par son frère? Nous ne pouvons le savoir car le roman s'arrête avant qu'Emmanuel n'ait atteint l'âge de la parole.

Devons-nous voir dans la disparition de Jean Le Maigre la perte du pouvoir créateur? Et sur le plan sociologique, un peuple qui perd son droit à la parole, qui n'a plus ses propres contes à dire, n'est-t-il pas un peuple qui perd son identité?

Le roman essaie d'effectuer le passage de la parole à une nouvelle génération. Y réussit-il? 